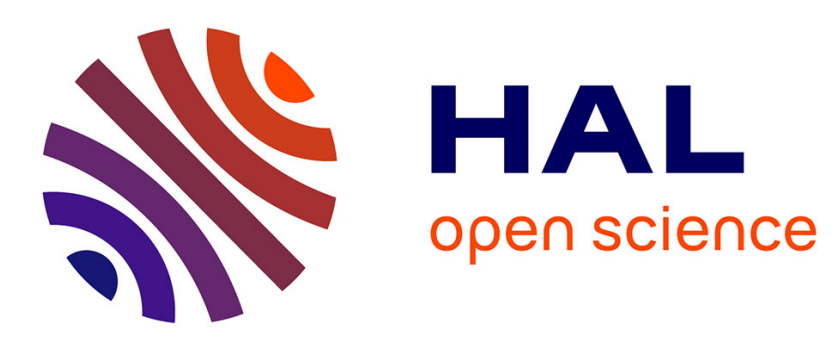

\title{
Possible Optimality and Preference Elicitation for Decision Making
}

\author{
Nawal Benabbou
}

\section{To cite this version:}

Nawal Benabbou. Possible Optimality and Preference Elicitation for Decision Making. The 4th International Conference on Algorithmic Decision Theory, Sep 2015, Lexington, United States. pp.561566, 10.1007/978-3-319-23114-3_34. hal-01217672

\section{HAL Id: hal-01217672 https://hal.sorbonne-universite.fr/hal-01217672}

Submitted on 22 Oct 2015

HAL is a multi-disciplinary open access archive for the deposit and dissemination of scientific research documents, whether they are published or not. The documents may come from teaching and research institutions in France or abroad, or from public or private research centers.
L'archive ouverte pluridisciplinaire HAL, est destinée au dépôt et à la diffusion de documents scientifiques de niveau recherche, publiés ou non, émanant des établissements d'enseignement et de recherche français ou étrangers, des laboratoires publics ou privés. 


\title{
Possible Optimality and Preference Elicitation for Decision Making
}

\author{
Nawal Benabbou \\ Sorbonne Universités, UPMC Univ Paris 06 \\ CNRS, LIP6 UMR 7606 \\ 4 Place Jussieu, 75005 Paris, France \\ nawal.benabbou@lip6.fr
}

Decision support systems often rely on a mathematical decision model allowing the comparison of alternatives and the selection of a proper solution. In the field of Multicriteria Decision Making, an aggregation function is often used to synthesize the different evaluations of each alternative into an aggregated value representing its overall utility. The aggregation function must be sufficiently expressive to efficiently approximate the decision maker's preferences in human decision support, or simulate a prescribed decision behavior in automated decision systems. This explains the diversity of decision models available in the literature but also the increasing interest for sophisticated parameterized models such as the Choquet integral $[32,14]$ which enables the representation of complex preferences and includes many other models as special cases (e.g. leximin and lexicographic aggregators [11], the Ordered Weighted Average operator [38], and Weighted Ordered Weighted Average [34]).

To make use of such models, one needs to assess the model parameters in order to fit to the decision maker's preferences. Most of the previous work on the elicitation of Choquet integral parameters consider a static database of preference statements, and focus on the determination of the parameters that best fit to the available database (e.g. $[16,26,27,13,15])$ for instance by minimizing a quadratic error. However, these approaches require a relatively large number of preference statements to model the decision maker's behaviour accurately which are not always possible to obtain. Preference elicitation with limited available information is a crucial task in many application domains, including recommender systems and interface customization [28]. Departing from these standard approaches, we consider incremental elicitation methods based on the minimax regret which is a decision criterion that has been advocated as a means for robust optimization in the presence of data uncertainty [21] and has been used for decision making with utility function uncertainty $[5,31,6]$. The general principle of this approach is to iteratively ask questions to the decision maker so as to reduce the set of possible parameters until the preferred alternative can be detected with some guarantees (as given by the minimax regret). This elicitation approach enables limiting the decision maker's burden as preference information is only required to discriminate between alternatives (not to assess the model parameters). Incremental elicitation methods have been proposed for the simple case of linear utilities but have never been studied for Choquet Integrals. This constitutes the first challenging issue considered during my $\mathrm{PhD}$. 
Incremental elicitation of Choquet Integrals. Adapting the incremental elicitation approach used for linear functions to the case of Choquet integrals is not straightforward. In the linear case, the selection of new preference queries requires multiple linear programs to be solved, but these problems become significantly more difficult when considering a Choquet integral. More precisely, the Choquet integral's parameters take the form of a capacity, i.e. a monotonic function defined on the power set of criteria which enables the control of the importance attached to all subsets of criteria, and possibly positive or negative synergies between criteria. Thus the number of parameters to assess is exponential in the number of criteria. As a consequence, the nature of the Choquet integral's parameters induces an exponential number of optimization variables and an exponential number of constraints over these variables in the linear programs considered in this elicitation scheme. First, we have proved that, by focusing on a specific type of queries involving binary profiles versus constant profiles, the optimization problems to be solved can be simplified to problems admitting only a linear number of variables and constraints. Then, using the constraint graph associated with these simplified linear programs, we have proposed an iterative procedure to solve these optimization problems in polynomial time (instead of using linear programming); our iterative procedure reduces computation times by around five orders of magnitude. Finally, we tested "the query selection strategy" which consists of selecting the question that is the most informative in the worst-case scenario of answers and we observed that it enables the detection of the preferred option without asking too many questions. This work has been accepted for publication in the last European Conference on Artificial Intelligence and honored to receive the ECAI'14 Best Student Paper award [4].

The next step was to consider decision situations where the decision space is very large, which is often the case in recommender systems where the possible alternatives can be thousands. Elicitation on combinatorial domains is a challenging issue that recently motivated several contributions in various contexts, e.g. in constraint satisfaction problems [6], in Markov Decision Processes $[30,36]$, in stable matching problems [10] and in multiattribute spaces [12, 7, 20]. We consider decision spaces defined implicitly as the set of possible solutions of a multiobjective combinatorial optimization problem. In standard interactive methods for multicriteria decision support, preference elicitation methods consists in iterating the generation of a feasible instance of the parameters and the computation of the corresponding optimal solution, until the decision maker is satisfied with the latter solution (e.g. [39, 35]). However, this elicitation approach does not guarantee that the final solution is actually the best alternative for the decision maker since the final instance of the model parameters does not necessarily fit to the decision maker's preferences. We proposed instead to combine search for possibly optimal solutions and elicitation in order to reduce the uncertainty over the model parameters during the resolution so as to more focus the search while determining a necessary optimal solution. A possibly (resp. necessary) optimal solution is an option that is optimal for some (resp. all) parameters compatible with our knowledge about the decision maker's preferences. We need 
first to propose efficient search procedures for the determination of all possibly optimal solutions given a set of feasible parameters, and then to design elicitation methods reducing the set of feasible parameters during the search while ensuring the determination of a necessary optimal alternative at the end of the resolution. So far, we have considered the two following multiobjective combinatorial optimization problems : multiobjective state space search and multicriteria spanning tree problem. In order to address one difficulty at a time, we have first considered the case of linear utilities and then Choquet Integrals.

Multiobjective State Space Search. Consider a state space graph endowed with $q$ evaluation criteria, i.e. $q$ cost functions to be minimized (e.g. time, distance, energy, risk). Each path is therefore valued by a cost vector and preferences over paths are inherited from the preference over their cost vectors. Preference over cost vectors are defined using a linear aggregation function $f_{\omega}$ defining the overall cost (or disutility) $f_{\omega}(x)$ attached to any cost vector $x$, where $\omega$ is a vector of preference parameters representing the relative importance of criteria. We want to find a path from an initial node to a goal node that minimizes the overall cost function $f_{\omega}$ that represents the DM's preferences, but the vector of weights $\omega$ is imprecisely known. Since all preference models considered in multicriteria analysis are compatible with Pareto dominance, preference-based search methods in multiobjective optimization are often based on the exploration of the set of Pareto-optimal solutions. The so-called MOA* algorithm [33, 25] is a multiobjective extension of $A^{*}$ [17] that determines the set of Pareto non-dominated cost vectors attached to solution paths and returns one path for each element. In the multiobjective case, there possibly exists several optimal paths with different cost vectors to reach a given node. Therefore, the basic graph exploration procedure consists in iteratively expanding subpaths rather than nodes. In order to compute the set of possibly optimal solution paths, we need to define new pruning rules that use sharper conditions than those based on Pareto-dominance tests. In other words, we need to be able to detect subpaths that cannot lead to possibly optimal solution given our knowledge about the decision maker's preferences. To do so, we introduced a dominance relation between sets of cost vectors and given a set of cost vectors, we proved that it enables to detect vectors that cannot be possibly optimal. Then, we proposed a filtering algorithm based on this dominance relation, enabling us to compute the set of possibly optimal cost vectors in a polynomial time. Finally, we proposed two pruning rules based on this filtering algorithm which enable us to discard subpaths that cannot lead to a possibly optimal solution path and we proved that the corresponding graph exploration procedure returns exactly the set of possibly optimal solution paths. Finally, to detect a necessary optimal solution paths, we proposed two incremental elicitation strategies based on the minimax regret criterion so as to reduce the uncertainty during the search. This work has been accepted for publication in the last AAAI Conference on Artificial Intelligence [2]. The next step was to extend our approach to work with a non-linear $f_{\omega}$ function such as a Choquet integral so as to obtain better fitting capacities to the decision maker's preferences. However, this extension raises challenging algorithmic questions since 
the use of a Choquet integral complicates the definition of pruning rules by not satisfying the Bellman principle (i.e. discarding supbaths by comparison with other subpaths is no longer possible). In order to design an efficient algorithm, we proposed to work on near optimal cost vectors using an approximate version of minimax regret. This work has been accepted for publication in the last IJCAI conference [1].

Multicriteria spanning tree problem. Consider a connected graph $G$ where each edge is valued by a cost vector corresponding to its evalutation with respect to different criteria. Every criterion is assumed to be additive over the edges; therefore the cost of any subgraph is the sum of the cost of its constituent edges. A spanning tree of $G$ is a connected subgraph of $G$ which contains no cycle while including every node of $G$. We assume that preferences over cost vectors are defined using a linear aggregation function $f_{\omega}$ but the weighting vector $\omega$ is imprecisely known. Here again, given a feasible set of weights, we first consider the problem of determining all possibly optimal spanning trees. In the single objective case, the minimum spanning tree problem can be solved in polynomial time using standard greedy algorithms due to Kruskal [22] and Prim [29]. Unfortunately, as soon as the number of criteria is greater than 2, the problem becomes intractable because the number of Pareto-optimal cost vectors associated to spanning trees is, in the worst case, exponential in the number nodes. In the paper "On Possibly Optimal Tradeoffs in Multicriteria Spanning Tree Problems" that has been accepted for publication in ADT 2015 [3], we proposed a multiobjective extension of Prim's algorithm which can compute the exact set of possibly optimal cost vectors associated to spanning trees; this algorithm is a greedy search based on a specific decomposition of the feasible set of parameters. Then, we proposed to interweave incremental elicitation and search to determine a necessary optimal spanning tree. This algorithm consists in selecting, at each iteration step, an edge in the cocycle of the current subgraph that is necessarily (or almost) optimal in the cocycle; if no such edge exists, the procedure asks questions to the decision maker to reduce the set of feasible weights until such edge can be detected. We are now studying the case of non-linear utility functions, which seems to be a challenging issue because our multiobjective greedy search is no longer valid.

Perspectives. As future work, we plan to study combinatorial voting with partial preference profiles. When individual preferences are incomplete, one can indeed study possible and necessary winners (e.g., $[19,37,23,9]$ ). In this setting, incremental elicitation methods are used to progressively reduce the set of possible winners until a winner can be determined with some guarantee $[18,24,8]$ ). As a next step, we can study the potential of incremental elicitation methods in combinatorial voting (i.e. the set of alternatives has a combinatorial structure) with partial preference profiles. We also plan to adapt these approaches for utility elicitation in the context of decision making under risk, not only for expected utility models but also for rank-dependent utility model (elicitation of the probability distortion combined with the elicitation of utilities). 


\section{References}

1. N. Benabbou and P Perny. Combining preference elicitation and search in multiobjective state-space graphs. In Proceedings of IJCAI'15, 2015.

2. N. Benabbou and P. Perny. Incremental Weight Elicitation for Multiobjective State Space Search. In Proceedings of AAAI'15, pages 1093-1098, 2015.

3. N. Benabbou and P. Perny. On possibly optimal tradeoffs in multicriteria spanning tree problems. In Proceedings of ADT'15, 2015.

4. N. Benabbou, P. Perny, and P. Viappiani. Incremental elicitation of Choquet capacities for multicriteria decision making. In Proceedings of ECAI'14, pages 87-92, 2014.

5. C. Boutilier, F. Bacchus, and R. I. Brafman. UCP-Networks: A directed graphical representation of conditional utilities. In Proceedings of UAI'01, pages 56-64, 2001.

6. C. Boutilier, R. Patrascu, P. Poupart, and D. Schuurmans. Constraint-based Optimization and Utility Elicitation using the Minimax Decision Criterion. Artifical Intelligence, 170(8-9):686-713, 2006.

7. D. Braziunas and C. Boutilier. Minimax regret based elicitation of generalized additive utilities. In Proceedings of UAI'07, pages 25-32, 2007.

8. L. Naamani Dery, M. Kalech, L. Rokach, and B. Shapira. Reaching a joint decision with minimal elicitation of voter preferences. Information Sciences, 278:466-487, 2014.

9. N. Ding and F. Lin. Voting with partial information: what questions to ask? In Proceedings of AAMAS'13, pages 1237-1238, 2013.

10. J. Drummond and C. Boutilier. Preference elicitation and interview minimization in stable matchings. In Proceedings of AAAI'14, pages 645-653, 2014.

11. P. C Fishburn. Axioms for lexicographic preferences. The Review of Economic Studies, pages 415-419, 1975.

12. C. Gonzales and P. Perny. GAI Networks for Utility Elicitation. In Proceedings of the 9th International Conference on the Principles of Knowledge Representation and Reasoning, pages 224-234, 2004.

13. M. Grabisch, I. Kojadinovic, and P. Meyer. A review of methods for capacity identification in Choquet integral based multi-attribute utility theory. European Journal of Operational Research, 186(2):766-785, 2008.

14. M. Grabisch and C. Labreuche. A decade of application of the Choquet and Sugeno integrals in multi-criteria decision aid. Annals of Operations Research, 175(1):247$286,2010$.

15. M. Grabisch, J.-L. Marichal, R. Mesiar, and E. Pap. Aggregation Functions. Encyclopedia of Mathematics and its Applications. Cambridge University Press, NewYork, 2009.

16. M. Grabisch, H. T. Nguyen, and E. A. Walker. Fundamentals of Uncertainty Calculi, with Applications. Encyclopedia of Mathematics and its Applications. Kluwer Academic Publishers, 1995.

17. P. E. Hart, N. J. Nilsson, and B. Raphael. A formal basis for the heuristic determination of minimum cost paths. IEEE Transactions on Systems, Man, and Cybernetics, 4(2):100-107, 1968.

18. M. Kalech, S. Kraus, and Gal A. Kaminka. Practical voting rules with partial information. Autonomous Agents and Multi-Agent Systems, 22(1):151-182, 2010.

19. K. Konczak and J. Lang. Voting procedures with incomplete preferences. In Proceedings of IJCAI-05 Multidisciplinary Workshop on Advances in Preference Handling, volume 20, 2005. 
20. F. Koriche and B. Zanuttini. Learning conditional preference networks. Artificial Intelligence, 174(11):685-703, 2010.

21. P. Kouvelis and G. Yu. Robust Discrete Optimization and Its Applications. Kluwer, Dordrecht, 1997.

22. J. B. Kruskal. On the shortest spanning subtree of a graph and the traveling salesman problem. Proceedings of the American Mathematical Society, 7:48-50, 1956.

23. J. Lang, M. S. Pini, F. Rossi, D. Salvagnin, K. Brent Venable, and T. Walsh. Winner determination in voting trees with incomplete preferences and weighted votes. Autonomous Agents and Multi-Agent Systems, 25(1):130-157, 2012.

24. T. Lu and C. Boutilier. Robust approximation and incremental elicitation in voting protocols. In Proceedings of IJCAI'11, pages 287-293, 2011.

25. L. Mandow and J. L. Pérez De la Cruz. A new approach to multiobjective A* search. In Proceedings of IJCAI'05, pages 218-223, 2005.

26. J.-L. Marichal and M. Roubens. Determination of weights of interacting criteria from a reference set. EJOR, 124(3):641-650, 2000.

27. P. Meyer and M. Roubens. On the use of the Choquet integral with fuzzy numbers in multiple criteria decision support. Fuzzy Sets and Systems, 157(7):927-938, 2006.

28. B. Peintner, P. Viappiani, and N. Yorke-Smith. Preferences in interactive systems: Technical challenges and case studies. AI Magazine, 29(4):13-24, 2008.

29. R. C. Prim. Shortest connection networks and some generalizations. Bell System Technical Journal, 36:1389-1401, 1957.

30. K. Regan and C. Boutilier. Eliciting additive reward functions for markov decision processes. In Proceedings of IJCAI'11, pages 2159-2164, 2011.

31. A. Salo and R. P. Hämäläinen. Preference ratios in multiattribute evaluation (PRIME)-elicitation and decision procedures under incomplete information. IEEE Trans. on Systems, Man and Cybernetics, 31(6):533-545, 2001.

32. D. Schmeidler. Integral representation without additivity. Proceedings of the American Mathematical Society, 97(2):255-261, 1986.

33. Bradley S. Stewart and Chelsea C. White III. Multiobjective A*. Journal of ACM, 38(4):775-814, 1991.

34. V. Torra. The weighted OWA operator. International Journal of Intelligent Systems, 12(2):153-166, 1997.

35. D. Vanderpooten and P. Vincke. Description and analysis of some representative interactive multicriteria procedures. Appl. Math. Comp., 83(2-3):261-280, May 1997.

36. P. Weng and B. Zanuttini. Interactive Value Iteration for Markov Decision Processes with Unknown Rewards. In Proceedings of IJCAI'13, pages 2415-2421, 2013.

37. L. Xia and V. Conitzer. Determining possible and necessary winners given partial orders. Journal of Artificial Intelligence Research (JAIR), 41:25-67, 2011.

38. R. R. Yager. On Ordered Weighted Averaging aggregation operators in multicriteria decision making. IEEE Trans. Systems, Man and Cybern., 18(1):183-190, 1998.

39. S. Zionts and J. Wallenius. An interactive programming method for solving the multiple criteria problem. Management Science, 22(6):652-663, 1976. 